\title{
The Big Billion Indian COVID 19 Vaccine Challenge
}

\author{
Arvind Krishnamurthy ${ }^{1} \cdot$ K. S. Gopinath ${ }^{2,3}$
}

Published online: 1 February 2021

(C) Indian Association of Surgical Oncology 2021

\section{"My advice to anyone offered the vaccine is to take it. If I can have it at 90 , then you can have it too." Margaret Keenan (First person in the world to have the Covid-19 vaccine on December $8^{\text {th }} 2020$ )}

Come 2021, COVID-19 vaccination is expected to be one of the most eagerly awaited events in the timelines of every nation world-wide and India is no exception to this mammoth global initiative. It is heartening to note the highly promising efficacy data from at least six vaccine makers i.e., PfizerBiontech, Moderna, Oxford-AstraZeneca, Gamaleya Center's Sputnik V vaccine, Novamax and the Johnson and Johnson vaccine. While the Pfizer-Biontech vaccine has claimed an efficacy of $95 \%$, the Moderna in its latest release has claimed an efficacy of $94 \%$. The adenovirus-based vaccine developed by Oxford- AstraZeneca has claimed an 70\% average efficacy, while the Russian Gamaleya Center's Sputnik V vaccine, another adenovirus-based vaccine, has reported an efficacy of $91 \%$. These results are expected to be formally published in peer reviewed journals soon. Further, many vaccine candidates are being developed at an accelerated pace and more than 180 such candidates have in fact reached the phase of clinical evaluation. It is commendable to note that barely a year since the emergence of the COVID19 pandemic, multiple vaccine developers have defied the traditional timelines of many years for vaccine development and are now poised to secure emergency use authorization [1].

Arvind Krishnamurthy

drarvindkrishnamurthy@yahoo.co.in

K. S. Gopinath

gopiijso@gmail.com

1 Surgical Oncology, Cancer Institute (WIA), 38, Sardar Patel Rd, Adyar, Chennai 600036, India

2 HCG Hospital, Bengaluru, India

3 Sri Devaraj Urs Medical College, Kolar, India
The front runner vaccine candidates for India's COVID-19 response seems to be the vaccines developed by OxfordAstraZeneca and the Bharath Biotech thus far. Although Pfizer India has formally approached the Drugs Controller General of India (DCGI) for EUA of its Covid-19 vaccine, there seems to be no clear roadmap for the availability of the mRNA vaccines developed by Pfizer as well as the Moderna in India till date. It must be noted that the specialized cold storage requirements for the above mRNA vaccines may be a deterrent to its large-scale use in India. This is a rapidly evolving field and clinical recommendations and policy decisions are expected to periodically change with the accumulation of more and more scientific evidences.

From the immunological stand-point there seems to be a lack of clarity as to what condition induces a stronger immune response i.e., a natural COVID-19 infection or following vaccine administration. Studies have reported a huge dynamic variation in immune responses to natural infected patients. The diversity in the immune responses to the natural infection can be partly explained by the differences in the amount of virus to which the contact was exposed to, among other factors. Experts are of the view that Covid-19 vaccination can predictably prevent illness and it undoubtedly seems to be a much safer bet. Hoping to get and subsequently recover from a Covid-19 infection clearly seems to be a far more dangerous option considering the unpredictable responses, severity of infection and the outcomes of a naturally acquired COVID19 infection. Further, nearly a third of the patients (including even younger patients with no comorbid conditions) who recover from COVID-19 infection seem to have chronic symptoms like exhaustion, tachycardia, symptoms similar to lupus or rheumatoid arthritis and even erectile dysfunction. The safety data of the various COVID-19 vaccines administered to many thousands of patients seem to suggest a favorable safety profile thus far with minimal serious side effects [1]. Preliminary reports further suggest that COVID-19 vaccination can augment the immune responses in patients who have recovered from a natural COVID-19 infection. It is important for the treating clinician to understand that the ongoing vaccine trials only test for the efficacy of the candidate vaccines, 
whereas the actual effectiveness of the vaccine will be known only when a large proportion of the population are vaccinated post-licensure. Further, given the novelty of COVID-19 infection, there are several outstanding issues that remain unaddressed which include the duration of the protection following vaccination and the long-term safety of the candidate vaccines.

It is noteworthy to mention that while developing a good vaccine candidate in itself is a herculean task, equally and perhaps more challenging for a 1.3 billion populous nation like India would be to effectively procure, transport and vaccinate its citizens in a planned and structured manner. It is interesting to note the Indian governments vaccine roll out plan for the nation. The initial focus seems to be on vaccinating the health care and the front-line workers, (critical elements of human resources to our COVID-19 response) followed by the age and risk-based vaccination strategy. We as oncologists must ensure that our patients get a reasonable priority in the vaccine roll out strategy considering their heightened vulnerability and a higher case fatality rate as compared to COVID-19 patients without cancer [2]. The mRNA vaccines are in fact speculated to be promising vaccine candidates for patients with solid cancers. The mRNAs in the vaccine are encapsulated into lipid carriers and the small liposomes are believed to accumulate in cancer tissues through the permeation retention effect [3]. It is vitally important for the scientific community to specifically ascertain the safety and efficacy data of the COVID-19 vaccines in cancer patients as some of the vaccine trials have excluded patients either with active cancer, on immunosuppressive treatments or in patients with a prior history of cancer.

What is perhaps very crucial for effective vaccination is the element of public trust in the vaccine enterprise initiative. The Government from its side must ensure a high level of transparency and scientific integrity in issuing advisories and in periodically addressing the public concerns. This trust factor will have a direct bearing on the willingness of the public to come forward for mass vaccination and more importantly their adherence to the needs of vaccination so as to reach the desired vaccination coverage. The increasing roles played by the Non-Governmental organizations, Media, (both print as well as digital) policy makers and health care professionals in percolating the messages of continued masking, hand washing and social distancing is well acknowledged. The Indian Journal of Surgical Oncology (IJSO) in its part, has published various scientific manuscripts pertaining to the management of cancer patients amidst the COVID-19 pandemic $[4,5]$.

We as a nation must ensure that every willing Indian citizen, as prioritized by the Government ultimately gets access to the vaccine, without any fear or favor and more importantly without any discrimination based on region, gender, religion or social status. It is also important for the scientific community as well as the policy makers to view the process of vaccination as a tool for promoting health equity and executing this initiative well will safeguard the health of our citizens as well as our economy.

We wish our readers a very happy, prosperous, healthy and a safe New Year 2021.

\section{References}

1. Mullard A (2020) COVID-19 vaccines buoy hope. Nat Rev Drug Discov. https://doi.org/10.1038/d41573-020-00215-9 Epub ahead of print

2. Zhang H, Han H, He T, Labbe KE, Hernandez AV, Chen H, Velcheti V, Stebbing J, Wong KK (2020) Clinical Characteristics and Outcomes of COVID-19-Infected Cancer Patients: A Systematic Review and Meta-Analysis. J Natl Cancer Inst 2:djaa168

3. Fanciullino R, Ciccolini J, Milano G (2020) COVID-19 vaccine race: watch your step for cancer patients. Br J Cancer. https://doi. org/10.1038/s41416-020-01219-3 Epub ahead of print

4. Krishnamurthy A, Gopinath KS (2020) The need for prioritizing Cancer surgeries amidst the COVID-19 pandemic. Indian J Surg Oncol. 29:1-2

5. Shrikhande SV, Chaudhary A, Krishnamurthy A, Rao GV, Chaturvedi H, Kar M et al (2020) Surgical oncology practice in the wake of COVID-19 crisis. Indian J Surg Oncol 7:1-7

Publisher's Note Springer Nature remains neutral with regard to jurisdictional claims in published maps and institutional affiliations. 